\title{
LAS ACTITUDES HACIA LOS INMIGRANTES EN MÉXICO: EXPLICACIONES ECONÓMICAS Y SOCIALES
}

Covadonga Meseguer

Gerardo Maldonado

\section{INTRODUCGIÓN}

MÉXICO NUNCA HA SIDO UN IMÁN DE LA INMIGRACIÓN, ni siquiera en la época en que la política de inmigración era liberal, a diferencia de otros países latinoamericanos en los que la inmigración fue un fenómeno masivo en el siglo xx (Argentina, Brasil, Chile o Uruguay, por ejemplo). Según el censo más reciente de 2010, aproximadamente un millón de extranjeros viven en México, lo que equivale a menos de $1 \%$ de la población del país. Sin embargo, de acuerdo con los resultados de la encuesta México, las Américas y el Mundo del mismo año, uno de cada tres mexicanos cree que hay muchos extranjeros viviendo en el país. Este fenómeno podría justificarse si hubiera una competencia en el mercado laboral o si los inmigrantes resultaran una amenaza para la seguridad del país o su relativa homogeneidad étnica. ¿Existe esa percepción?

Para responder a esta pregunta exploramos los factores determinantes de las actitudes individuales hacia la inmigración en México. Considerando que este tipo de actitudes ha sido ampliamente analizado en los países avanzados, ${ }^{1}$ este es, hasta donde sabemos,

${ }^{1}$ Jack Citrin, Donald Green, Christopher Muste y Cara Wong, "Public Opinion toward Immigration Reform: The Role of Economic Motivations", Journal of Politics, vol. 59, 1997; Joel Fetzer, Public Attitudes toward Immigration in the United States, France, and Germany, Nueva York, Cambridge University Press, 2000; Charles Chandler y Yung-mei Tsai, "Social Factors Influencing Immigration Attitudes: An 
uno de los primeros trabajos que estudia las causas de las actitudes individuales hacia los extranjeros en países con mano de obra abundante y países de emigración y de transmigración. ${ }^{2}$ México merece una atención especial, en tanto los dos principales grupos de inmigrantes (estadounidenses y guatemaltecos) son radicalmente diferentes en términos de cualificaciones, rasgos étnicos y causas de migración. México también es una ruta muy importante para los trans-migrantes centroamericanos y sudamericanos en su camino a los Estados Unidos.

Para analizar este tema utilizamos la edición 2010 de la encuesta México, las Américas y el Mundo, ${ }^{3}$ y proponemos dos hipótesis generales. Por un lado, exploramos si los intereses materiales explican las distintas opiniones hacia los extranjeros. Las razones económicas de la migración anticipan un efecto distributivo de la inmigración en los países receptores. Según estos modelos, las personas cuyos salarios, ingresos y perspectivas de empleo se ven amenazados por la llegada de extranjeros suelen apoyar políticas restrictivas y tienen opiniones menos entusiastas sobre los inmigrantes. En pocas palabras, los individuos con capacidades o habilidades

Analysis of Data from the General Social Survey", Social Science Journal, vol. 38, núm. 2, 2001, pp. 177-188; Kenneth Scheve y Matthew Slaughter, "Labor Market Competition and Individual Preferences over Immigration Policy", The Review of Economics and Statistics, vol. 83, núm 1, 2001; Lauren M. McLaren, "Anti-Immigrant Prejudice in Europe: Contact, Threat Perception, and Preferences for the Exclusion of Migrants", Social Forces, vol. 81, núm 3, 2003; Anna Maria Mayda, "Who Is Against Immigration? A Cross-Country Investigation of Individual Attitudes toward Immigrants", The Review of Economics and Statistics, vol. 88, 2006; John Sides y Jack Citrin, "European Opinion about Immigration: The Role of Identities, Interests, and Information", British Journal of Political Science, vol. 37, 2007; Jack Citrin y John Sides, "Immigration and the Imagined Community in Europe and the United States", Political Studies, vol. 56, 2008; Jens Hainmueller y Michael Hiscox, "Educated Preferences: Explaining Attitudes toward Immigration in Europe", International Organization, vol. 61, 2007; Jens Hainmueller y Michael Hiscox, "Attitudes toward Highly Skilled and Low-Skilled Immigration: Evidence from a Survey Experiment", American Political Science Review, vol. 104, 2010.

2 Diana M. Orcés, "Democratic Values and Public Opinion toward Immigrants: The Case of Ecuador", Latin American Politics and Society, vol. 51, núm. 4, 2009, pp. 131-155.

${ }^{3}$ México, las Américas y el Mundo, México, CIDE, 2010. 
(skills) similares a las de los inmigrantes deberían oponerse a la flexibilización de las restricciones migratorias. Por el otro, la hipótesis alternativa sostiene que los factores sociales, no económicos, tales como el orgullo nacional, la preocupación por la inseguridad y la intolerancia a ideas extranjeras determinan cómo los individuos consideran a los extranjeros y cómo valoran la llegada de inmigrantes. Como mostramos, tanto las preocupaciones materiales por el mercado de trabajo como las preocupaciones etnocéntricas han cambiado la política de inmigración en México durante el siglo $\mathrm{xx} .^{4}$

Nuestros resultados fueron los siguientes. En primer lugar, los temores de competencia en el mercado laboral tienen poco poder explicativo en la consideración de los mexicanos por los extranjeros. Usando varias operacionalizaciones de los niveles de capacidades (skills) de los individuos (años de escolaridad y habilidades profesionales), así como su situación laboral (dentro y fuera del mercado laboral), encontramos que el interés individual material está relacionado de forma débil con las opiniones sobre los inmigrantes. Cuando los intereses individuales importan, es en la manera contraria a lo esperado por las teorías distributivas de inmigración. Las consideraciones económicas importan sólo de una manera socio-trópica: pensar que los extranjeros contribuyen a la economía se relaciona positivamente con mantener una mejor opinión de los inmigrantes.

Dada la visibilidad de los recientes acontecimientos relacionados con la seguridad de los migrantes centroamericanos y de los abusos a los derechos humanos de los trans-migrantes en su camino hacia los Estados Unidos, se tomaron en cuenta los problemas

${ }^{4}$ La encuesta México, las Américas y el Mundo es una herramienta única para comprobar si estos dos tipos de preocupaciones (económicas y sociales) dan forma a las opiniones que tienen los mexicanos sobre los inmigrantes en general, los estadounidenses y guatemaltecos en particular. Si bien existen otras encuestas de opinión pública en México que tienen información sobre las opiniones hacia los extranjeros, como las realizadas por el Consejo Nacional para Prevenir la Discriminación (Conapred) o el Centro de Estudios Sociales y de Opinión Pública (CESOP) de la Cámara de Diputados, estas encuestas no cuentan con las preguntas necesarias para poner a prueba nuestros argumentos. 
de seguridad. ${ }^{5}$ En ese sentido hallamos que aquellos que opinan que los migrantes amenazan la seguridad tienden a percibir que los extranjeros son muchos y a tener peores opiniones de los inmigrantes.

$\mathrm{El}$ artículo procede de la siguiente manera. En la primera sección se revisan las diferentes teorías de distribución de la migración para identificar a las personas que se espera que estén a favor y en contra de la inmigración de acuerdo con quién gana y quién pierde con la inmigración. En la sección 2 se describen las características relevantes de los inmigrantes en México: principales grupos, sus niveles de habilidades y ocupaciones. En la sección 3 repasamos brevemente la política de inmigración de México desde una perspectiva histórica. En esta sección se muestra cómo una fuerte ideología nacionalista ha definido la manera en que la política de inmigración ha evolucionado a lo largo del tiempo. En la sección 4 presentamos los datos y modelos. En la sección 5 se discuten los resultados. Y llegamos a la conclusión en la sección 6 con una discusión de cómo estos resultados se relacionan con el conocimiento actual de los factores determinantes en las actitudes hacia los inmigrantes y sus implicaciones para algunos aspecto de la política migratoria actual.

\section{Preocupaciones económicas: competencia en el mercado LABORAL E INMIGRACIÓN CONTEMPORÁNEA EN MÉXICO}

La economía política considera que la opinión pública hacia los inmigrantes pone el énfasis en los temores por el mercado de trabajo. El modelo más destacado para anticipar quién gana y quién pierde con la inmigración es el modelo de economía cerrada de Factor-Proporciones (FP). ${ }^{6}$ Este modelo deriva las consecuencias

${ }^{5}$ Por ejemplo, en San Fernando, Tamaulipas, 72 transmigrantes fueron asesinados en agosto de 2010. Esto hizo evidente que los transmigrantes son frecuentemente presa del crimen organizado y del narcotráfico. No obstante estos migrantes fueron evidentemente víctimas, la opinión pública asocia la presencia de inmigración con un empeoramiento del crimen. Para esto véase Informe especial sobre secuestro de migrantes en México, CNDH, 2011.

${ }^{6}$ George Borjas, Heaven's Door: Immigration Policy and the American Economy, 
distributivas de la inmigración al concentrarse en cómo la inmigración altera el suministro de factores de producción en el país de destino. El dato fundamental es la composición de habilidades (skills) de los ciudadanos nacionales respecto a la composición de habilidades de los inmigrantes. Si los inmigrantes están menos capacitados que los ciudadanos del país receptor, los nacionales poco calificados pierden y los nacionales altamente calificados ganan con el aumento de la inmigración. Lo contrario ocurre si los inmigrantes son relativamente más calificados que los ciudadanos nacionales. En general, la predicción del modelo FP es sencillo: los ciudadanos del país receptor deberían oponerse a la llegada de inmigrantes con niveles de capacidades similares y apoyar la llegada de inmigrantes con habilidades distintas.

Sin embargo, las pruebas empíricas sobre qué tan bien las expectativas de distribución previstas en el modelo de FP explican las actitudes de los individuos hacia los inmigrantes no son concluyentes y encuentran resultados ambivalentes. Por un lado, los artículos de Scheve y Slaughter ${ }^{7}$ y de Mayda ${ }^{8}$ reportan resultados que dan un fuerte apoyo al modelo FP. Mayda ${ }^{9}$ encuentra que en los países avanzados las actitudes pro-inmigración correlacionan positivamente con mayores habilidades, mientras que en los países en desarrollo ocurre lo contrario: las actitudes pro-inmigración correlacionan negativamente con los niveles de habilidad menores. La explicación es que los países avanzados atraen inmigrantes que son relativamente menos cualificados que los nacionales, en contraste con los países en desarrollo que atraen inmigrantes que son relativamente más cualificados que los nativos. ${ }^{10}$

Princeton, Princeton University Press, 1999; George Borjas, Richard Freeman y Lawrence Katz, "Searching for the Effect of Immigration on the Labor Market", American Economic Review, vol. 86, núm. 2, 1996; George Borjas, Richard Freeman y Lawrence Katz, "How Much Do Immigration and Trade Affect Labor Market Outcomes?”, Brooking Papers on Economic Activity, vol. 1, 1997.

${ }^{7}$ Scheve y Slaughter, art. cit.

${ }^{8}$ Mayda, art. cit.

${ }^{9}$ Loc. cit.

${ }^{10}$ De hecho, Mayda encuentra que "cualquier país con el PIB per cápita en 1995 (ajustado por PPA) inferior a aproximadamente 4480 dólares se caracteriza 
Por el otro lado, Hainmueller y Hiscox ${ }^{11}$ encuentran resultados distintos. Primero, en su estudio con base en los datos de la Encuesta Social Europea, las preguntas acerca de los inmigrantes se refinaron para permitir distinguir entre las actitudes hacia inmigrantes altamente calificados e inmigrantes poco calificados. Contrario a las predicciones del modelo FP, los autores encuentran que las personas con nivel educativo superior y mayores habilidades profesionales son más propensas a favorecer la inmigración, prescindiendo de los niveles de cualificación de los inmigrantes. Segundo, los resultados de su trabajo con un diseño experimental dentro de una encuesta son similares. Hallan que tanto los ciudadanos nacionales poco calificados como los altamente calificados prefieren inmigrantes altamente calificados. Este resultado pone en tela de juicio el papel de la competencia en el mercado laboral y los intereses materiales en la formación de actitudes individuales hacia los inmigrantes.

Una limitación importante de estos estudios es que exploran casi en exclusivo las actitudes hacia inmigrantes en los países avanzados (Mayda es la excepción). La falta de disponibilidad de datos comparables entre países sobre flujos de poblaciones de inmigrantes y niveles de cualificación en relación con la población nacional es, por supuesto, un problema importante para explorar el impacto de la inmigración en los mercados laborales de los países menos desarrollados, donde el trabajo es un factor abundante. Además, el conocimiento sobre el efecto de la inmigración en salarios, ingreso y empleo en los países en desarrollo es prácticamente inexistente. ${ }^{12}$

por una correlación negativa entre la habilidad y las preferencias pro-inmigración, mientras que los individuos procedentes de países con PIB per cápita por encima de este umbral tienen mayor probabilidad de estar a favor de la inmigración conforme al aumento en el nivel educativo" (énfasis añadido, p. 519).

${ }^{11}$ Hainmuller y Hiscox, art. cit.

${ }^{12} \mathrm{Al}$ respecto existen dos estudios recientes que reportan resultados diferentes. Por un lado, Tim Gindling ("South-South Migration: The Impact of Nicaraguan Immigrants on Earnings, Inequality and Poverty in Costa Rica”, World Development, vol. 37, núm. 1, 2009) estudia el caso de Costa Rica, y encuentra que los inmigrantes nicaragüenses tuvieron un efecto negativo sobre los ingresos de 
Por desgracia, hay escasez de datos en las encuestas comparativas acerca de la política de inmigración y los inmigrantes en países de economías emergentes y en desarrollo. ${ }^{13}$ Esto resulta sorprendente e injustificado dado que $50 \%$ de los inmigrantes se mueven Sur-Sur y que, por lo tanto, la inmigración es una cuestión relevante en algunas regiones en desarrollo. ${ }^{14}$ Facchini, Mayda y Mendola ${ }^{15}$ han empezado a llenar este espacio usando tres rondas de la Encuesta Mundial de Valores (wvs) en Sudáfrica. Ellos encontraron que el mercado laboral no juega un rol importante en la formación de preferencias públicas acerca de inmigración, a pesar

mujeres costarricenses con menores niveles educativos. Este es un caso de migración Sur-Sur que se parece a lo que se sabe sobre la migración Sur-Norte: los nicaragüenses menos calificados afectan a los ingresos de los costarricenses menos calificados, sesgados por el género. Por otro, el estudio de Giovanni Facchini, Anna Maria Mayda y Mariapia Mendola ("South-South Migration and the Labor Market: Evidence from South Africa”, documento presentado en la $8^{\text {th }}$ IZA Annual Migration Meeting and 3rd Migration Topic Week, Washington, 12-15 de mayo de 2011) sobre la inmigración en Sudáfrica muestra que este país se ha convertido en un destino atractivo para los trabajadores altamente cualificados, por lo que parece afectar las perspectivas de empleo de sudafricanos altamente calificados. Aunque no se ha estudiado la opinión pública respecto a la inmigración, en Costa Rica es de esperar que los ciudadanos nacionales poco cualificados (especialmente las mujeres) se opongan a los inmigrantes, mientras que en Sudáfrica se podría anticipar que los nacionales altamente calificados se opondrían así a la inmigración. Incluso un tema tan prominente como la emigración de mexicanos y sus consecuencias en el mercado laboral de México tampoco ha sido estudiado. En un texto reciente, Prachi Misha ("Emigration and Wages in Source Countries: Evidence from Mexico", Journal of Development Economics, vol. 82, 2007) encuentra que la emigración a los Estados Unidos entre 1970 y 2000 ha producido un incremento en los ingresos promedio de los trabajadores mexicanos de alrededor de $8 \%$. Además, aquellos más beneficiados por la migración han sido los sectores con mejores niveles de educación (aquellos entre 12 y 15 años de instrucción). Así, la emigración parece contribuir al incremento en la desigualdad de ingresos en México.

13 Alin Ceobanu y Xavier Escandell, "Comparative Analysis of Public Attitudes Toward Immigrants and Immigration Using Multinational Survey Data: A Review of Theories and Research”, Annual Review of Sociology, vol. 36, 2010.

14 Dilip Ratha y William Shaw, "South-South Migration and Remittances", World Bank Working Paper, núm. 102, 2007.

${ }^{15}$ Facchini, Mayda y Mendola, "What Drives Attitudes towards Immigrants in South Africa?". 
del impacto de los inmigrantes en mercados laborales locales. Como mostramos más abajo, nuestros resultados para México sugieren una historia algo similar.

\section{INMIGRACIÓN CONTEMPORÁNEA A MÉXICO}

De acuerdo con la teoría económica, los nativos que comparten las mismas cualificaciones que los inmigrantes deberían oponerse a la inmigración. Lo contrario aplica para aquellos nativos con cualificaciones diferentes. Por esta razón, la prueba de hipótesis del mercado laboral debe comenzar con una revisión de los niveles de habilidades y ocupaciones de los migrantes que llegan a los países de destino. Cabe preguntarnos entonces ¿quién migra a México? Como mencionamos antes, la inmigración a los países en desarrollo es un tema inexplorado y los datos nacionales comparables no están disponibles con mucha facilidad. Esta es la situación de México, donde otro factor de complicación es que el interés en la inmigración es menor en comparación con la magnitud del fenómeno de emigración.

Como se muestra debajo, la característica de México como un país de emigración condiciona quiénes son los inmigrantes. De acuerdo con el censo 2010, más de $70 \%$ de los extranjeros que viven en México reportan haber nacido en los Estados Unidos. No obstante, más del $70 \%$ de estos individuos son niños menores de quince años que viven en familias donde el resto de los miembros se identifica como mexicanos. ${ }^{16}$ Además, más de un tercio de esos niños viven en municipios ubicados en la frontera norte de México y el otro tercio en estados con alta intensidad migratoria. La otra peculiaridad bien conocida de este grupo es que hay un número voluminoso de pensionados con alta movilidad que no se registra como inmigrantes legales y que mantienen una vida binacional. Después de los ciudadanos estadounidenses, los dos grupos más

${ }^{16}$ Ernesto Rodríguez, "La inmigración en México a inicios del siglo xx", en Ernesto Rodríguez (ed.), Extranjeros en México. Continuidades y aproximaciones, México, Centro de Estudios Migratorios, 2010. 
numerosos son guatemaltecos y españoles. Sin embargo, su presencia es seis veces menor que la de los estadounidenses. Este último es el grupo más numeroso, incluso después de ajustar por los niños menores de quince años: una vez que se depuran los datos, $50 \%$ de los inmigrantes caen en el intervalo de los 20 a los 44 años de edad.

En general, los extranjeros en México se asemejan en algo al patrón previsto por Mayda: ${ }^{17}$ en promedio, los inmigrantes están más cualificados que los nacionales. Entre los que tienen 25 años de edad y más, $53 \%$ tiene 13 años o más de educación. Entre 80 y $85 \%$ ha terminado la escuela secundaria y $42 \%$ tiene educación universitaria o más. La cifra anterior contrasta con el nivel educativo de los mexicanos: sólo el 12\% cuenta con educación universitaria o más. Al considerar esta estructura educativa, no es extraño que entre los inmigrantes, $37 \%$ ocupe puestos gerenciales o profesionales y $15 \%$ trabaje como técnicos o en labores administrativas. Según el Instituto Nacional de Estadística y Geografía (INEGI), un alto porcentaje $(65.7 \%)$ de los inmigrantes trabaja en el sector de servicios.

Una mirada más a fondo en los dos principales grupos de inmigrantes -estadounidenses y guatemaltecos- ofrece una mejor perspectiva de la complejidad de la inmigración a México. A diferencia de los inmigrantes en conjunto, y contrario a lo que cabría esperar, aquellos que reportan haber nacido en los Estados Unidos muestran niveles medios de logros educativos. Sólo 37\% alcanza educación secundaria. Además, los estadounidenses realizan una amplia gama de actividades. Hay varios datos interesantes que revela el Censo. En primer lugar, la estructura ocupacional de los estadounidenses que viven en la ciudad de México se parece a la estructura ocupacional de la comunidad inmigrante en general: los profesionales y directivos constituyen el mayor porcentaje (22\%). Sin embargo, sólo $9 \%$ del total de ciudadanos estadounidenses que viven en México son profesionales y directivos. En general, los ciudadanos estadounidenses en México presentan características muy peculiares que se relacionan con la larga historia de emigración

${ }^{17}$ Mayda, art. cit. 
masiva entre México y Estados Unidos: 1) más de la mitad de estos ciudadanos son menores de quince años, 2) su nivel de instrucción, y por lo tanto su estructura ocupacional, muestra una mayor variabilidad y 3) presentan menores tasas de participación laboral que los inmigrantes europeos y sudamericanos.

Los inmigrantes guatemaltecos en México son también un grupo peculiar. Los inmigrantes procedentes de Guatemala presentan bajos niveles de cualificación. Sólo 39\% de aquellos con 25 años o más completaron la escuela primaria y otro $39 \%$ no sabe leer ni escribir. La proporción de aquellos con educación secundaria o más es de sólo $12 \% .{ }^{18}$ A diferencia de otros grupos de inmigrantes, $30 \%$ de guatemaltecos trabaja en la agricultura y otro $30 \%$ se reporta como trabajadores por cuenta propia, concentrados en el sector informal. En conjunto, cerca de $80 \%$ de los inmigrantes de esta nacionalidad trabaja en empleos con bajo nivel de cualificación y la mitad de ellos corresponden a empleos en la agricultura. Los inmigrantes guatemaltecos se concentran principalmente en Chiapas y tienen una vida binacional en torno a la frontera sur. Sólo $5 \%$ de los guatemaltecos trabajan como profesionales y técnicos. Finalmente, los guatemaltecos son el grupo con la mayor proporción de individuos que hablan una lengua indígena de todos los extranjeros con residencia en México.

Con este panorama general, formulamos hipótesis acerca de cómo los temores de la competencia en el mercado laboral pueden dar forma a las actitudes de los mexicanos hacia los inmigrantes.

Hipótesis 1 (educación): Teniendo en cuenta que el inmigrante promedio en México está más capacitado que el mexicano promedio, se espera que los individuos más educados mantengan peores opiniones sobre los extranjeros y que sean más propensos a pensar que hay demasiados inmigrantes en el pais. (Este es un contraste interesante en comparación con las economías avanzadas.) Además, en términos generales, debería observarse el mismo patrón respecto a los

18 Guillermo Paredes, "Migración de guatemaltecos a México y Estados Unidos a partir de la Encuesta sobre Migración en la Frontera Guatemala-México 2004: Un análisis de estrategias migratorias”, Migraciones Internacionales, vol. 5, núm. 1, 2009. 
inmigrantes estadounidenses, pero a la inversa en el caso de los guatemaltecos.

Hipótesis 2 (situación en el mercado laboral): Esperamos que los individuos en el mercado laboral tengan peores opiniones de los inmigrantes, que aquellos que no están activos en el mercado laboral y que, por lo tanto, no compiten con los inmigrantes.

Hipótesis 3 (situación económica): Esperamos que los individuos que tienen una situación económica más favorable tengan menos miedo de la competencia en el mercado laboral. En consecuencia, ellos deben tener mejores opiniones sobre los inmigrantes que los que juzgan su situación económica menos favorable.

\section{Preocupaciones Sociales: XeNOFobia, XeNOfilia Y LAS IDENTIDADES}

Como mencionamos al inicio, México nunca ha sido un país de inmigración. El país apenas recibió un 3\% de los flujos transatlánticos de los inmigrantes que llegaron a América Latina en los albores del siglo xx. ${ }^{19}$ Incluso en los periodos más permisivos y liberales, la tasa de inmigrantes nunca superó el $1 \% .{ }^{20} \mathrm{Y}$ aunque, como veremos, la política de inmigración se dispuso a atraer personas altamente calificadas (en especial hombres) de orígenes europeo occidental y americano, México nunca los atrajo en gran número. ${ }^{21}$

El estudio de la inmigración mexicana ha sido bien documentado por historiadores. ${ }^{22}$ Los estudiosos suelen distinguir tres

${ }^{19}$ Delia Salazar, "Introducción”, en Delia Salazar (ed.), Xenofobia y xenofilia en la historia de México, siglos XIX y XX, México, Instituto Nacional de Migración/Instituto Nacional de Antropología e Historia/DGE Ediciones, 2006.

${ }^{20}$ Rodríguez, op. cit.

${ }^{21}$ Moisés González Navarro, Los extranjeros en México y los mexicanos en el extranjero, 1821-1970, vol. 1, México, El Colegio de México, 1993; Delia Salazar, "Tres momentos de la inmigración internacional en México, 1880-1946”, en Ernesto Rodríguez (ed.), Extranjeros en México: Continuidades y aproximaciones, México, Instituto Nacional de Migración-Dge Ediciones, 2010.

${ }^{22}$ Salazar, "Introducción"; Pablo Yankelevich, ¿Deseables o inconvenientes? Las fronteras de la extranjería en el México posrevolucionario, México, Bonilla Artigas/ ENAH/Iberoamericana Vervuert, 2011. 
periodos diferentes. El primer periodo coincide con el gobierno del general Porfirio Díaz (1877-1910). Este fue un periodo de apertura y de políticas activas para atraer a inmigrantes; sin embargo, a pesar de esta legislación amigable, llegaron pocos. El segundo periodo comprende los años posteriores a la revolución (1917-1974), cuando la hostilidad hacia los extranjeros se hizo evidente, especialmente en relación con ciertos grupos y nacionalidades, como los asiáticos. Por último, el tercer periodo va aproximadamente de 1974 a 1990, el cual inicia y termina con dos leyes de población. La Ley de 1974 coincide con la decadencia del modelo de industrialización que caracterizó al periodo anterior. El control demográfico resultó ser una prioridad. Desde el punto de vista de la inmigración, sólo las personas altamente calificadas (técnicos, científicos, administradores) eran bienvenidos. Tal como establecía el artículo 32, los inmigrantes debían ser "elementos útiles" para el país y tener los ingresos necesarios para su subsistencia. Considerando que persistió el énfasis en la necesidad de la protección de los empleos para los mexicanos, la asimilación y la identificación cultural de los extranjeros se diluyeron dentro de esta nueva ley. El otro acontecimiento importante que tuvo lugar durante este periodo es la llegada, una vez más, de grupos de refugiados que huyeron de los conflictos de la guerra civil en Guatemala y El Salvador y de los regímenes dictatoriales en el Cono Sur del continente. La Ley de Población de 1990 tardíamente tomó en cuenta este fenómeno con la regulación explícita de la condición de refugiado.

Entre 2010 y 2011, se discutió y aprobó una nueva Ley de Migración bajo la iniciativa del Partido Acción Nacional (PAN). Desde el punto de vista de la política de inmigración, no hubo grandes cambios: los inmigrantes altamente cualificados son especialmente bienvenidos, aunque con restricciones, a la vez que los procesos para obtener la condición de inmigrante legal y para obtener la ciudadanía mexicana se simplificaron. En la nueva Ley de Migración, los cambios más importantes están en el capítulo de sanciones, con el objetivo de alinear las demandas a las autoridades mexicanas con respecto al trato que reciben los emigrantes mexicanos con 
aquellas de los inmigrantes y transmigrantes en México. ${ }^{23}$ Por lo que hace a la posibilidad de atracción de migración calificada, la Ley de Migración busca de manera explícita proteger el mercado laboral nacional por medio de un sistema de cuotas y de apertura de empleo a extranjeros de forma ordenada y segura; sin embargo, a la fecha este sistema no ha sido implemantado. ${ }^{24}$

Es imposible entender la postura altamente selectiva hacia la llegada de extranjeros sin tomar en cuenta el papel del nacionalismo revolucionario en la conformación del proceso de construcción nacional. De hecho, este proceso tuvo como piedra angular la reafirmación del país contra la intervención de las sucesivas potencias extranjeras. Este fuerte sentimiento nacionalista echó raíces en el siglo xix entre la élite criolla que llevó a la independencia mexicana de España. Se expandió a las masas en el siglo xx, alimentado por la Revolución mexicana y las políticas sociales, la educación y

${ }^{23}$ La nueva Ley de Migración no sustituye la Ley de Población. En términos generales, la Ley reconoce la vulnerabilidad de los grupos transmigrantes en México, previene a las autoridades sobre su protección, ratifica la descriminalización del estatus de inmigración ilegal y le da estatus legal al Instituto Nacional de Migración (INM) que no había sido reconocido antes.

${ }^{24}$ El artículo 18 de la Ley de Migración establece entre las atribuciones de la Secretaría de Gobernación: "II. Fijar las cuotas, requisitos o procedimientos para la emisión de visas y la autorización de condiciones de estancia, siempre que de ellas se desprenda para su titular la posibilidad de realizar actividades a cambio de una remuneración; así como determinar los municipios o entidades federativas que conforman las regiones fronterizas o aquellas que reciben trabajadores temporales y la vigencia correspondiente de las autorizaciones para la condición de estancia expedida en esas regiones, en los términos de la presente Ley. En todos estos supuestos, la secretaría deberá obtener previamente la opinión favorable de la Secretaría del Trabajo y Previsión Social y tomará en cuenta la opinión de las demás autoridades que al efecto se establezcan en el Reglamento". Y en el artículo 58 de esta Ley se prevé el establecimiento de un "sistema de puntos" para determinar si un extranjero puede obtener la residencia permanente -sin necesidad de esperar los cuatro años de residencia previa que contempla la Ley en otras situacionesdonde se establece como un mínimo considerar: "II. Las capacidades del solicitante tomando en cuenta entre otros aspectos el nivel educativo; la experiencia laboral; las aptitudes en áreas relacionadas con el desarrollo de la ciencia y la tecnología; los reconocimientos internacionales, así como las aptitudes para desarrollar actividades que requiera el país". 
las políticas económicas de izquierda del PRI. La cercanía a los Estados Unidos y la fuerte asimetría de poder entre los dos países vecinos, así como momentos traumáticos de intervención en el pasado, alimentaron un sentimiento nacionalista de defensa del territorio y de los recursos naturales, y excluyente cuando se trata de una posible intervención extranjera en los asuntos políticos. Probablemente, los epítomes de este sentimiento fueron la nacionalización de la industria petrolera en 1936 y la consagración en la Constitución Mexicana de la prohibición para los extranjeros de intervenir en la política interna. ${ }^{25}$ La inmigración se concibe como un asunto de seguridad nacional.

Así, cuando se analiza desde una perspectiva histórica, las preocupaciones etno-céntricas y nacionalistas han sido fundamentales para el diseño de la política de inmigración en México. A pesar de que la estadística descriptiva sugiere que estas preocupaciones permanecen todavía, en este trabajo buscamos comprobar si estos rasgos tienen influencia en la configuración de los puntos de vista sobre los diferentes grupos de inmigrantes. En consecuencia, derivamos las siguientes hipótesis sobre aspectos relativos a identidades políticas y actitudes sociales:

Hipótesis 4 (nacionalismo): Se espera que los encuestados que declaran sentir un fuerte orgullo nacional tengan una peor opinión de (todos los grupos) inmigrantes y que crean que los extranjeros son muchos.

Hipótesis 5 (cosmopolitismo): Los encuestados que refieren opiniones positivas sobre la llegada de nuevas ideas y costumbres procedentes del extranjero tendrán una mejor opinión de (todos los grupos) inmigrantes y será más probable que piensen que hay pocos inmigrantes en México.

Hipótesis 6 (seguridad): Los temores de invasión e intervención sin duda han moldeado restricciones a la inmigración a lo largo

25 Enrique Florescano, Historia de las historias de la nación mexicana, México, Taurus, 2002; Agustín F. Basave, Análisis del nacionalismo mexicano en torno a la mestizofilia de Andrés Molina Enríquez, México, FCE, 2002; Lorenzo Meyer, "Estados Unidos y la evolución del nacionalismo defensivo mexicano", Foro Internacional, vol. 46, núm 3, 2006; Alan Knight, "Peasants into Patriots: Thoughts on the Making of the Mexican Nation”, Mexican Studies/Estudios Mexicanos, vol. 10, núm. 1, 1994. Yankelevich, op. cit. 
del tiempo. Pero hoy los temores de seguridad se relacionan con la presencia de grupos criminales que extorsionan a los migrantes en las fronteras norte y sur. En consecuencia, los encuestados que consideran a los inmigrantes como una amenaza a la seguridad deberían tener peor opinión de ellos y ser menos propensos a pensar que hay pocos inmigrantes.

Con toda la información anterior disponible, nosotros anticipamos que las preocupaciones sociales tendrán un mayor peso que las económicas al momento de configurar la perspectiva respecto de los inmigrantes en México. Cuando se evalúa la hipótesis de competencia en el mercado laboral, y después de considerar la limpieza de datos apropiada y la contabilización para un número sustancial de niños que aparecen en el censo como ciudadanos estadounidenses, debemos tener en mente que los inmigrantes en México pueden ser muy pocos para amenazar los salarios o perspectivas de trabajo de los mexicanos. Si este es el caso, debemos encontrar que la educación de los encuestados, su ocupación o estatus de mercado laboral explican poco las actitudes hacia la inmigración o la percepción sobre el número de inmigrantes.

\section{Datos y modelos}

Para poner a prueba nuestras hipótesis, utilizamos la encuesta $M e ́-$ xico, las Américas y el Mundo. ${ }^{26}$ Llevada a cabo cada dos años, esta es una encuesta de opinión pública sobre asuntos internacionales con muestras de población nacional y líderes en México y otros países de América Latina. Por primera vez, la edición 2010 de la encuesta incluyó una serie importante de preguntas acerca de la emigración y la inmigración. ${ }^{27}$ El cuestionario se aplicó cara a cara en viviendas a una muestra de la población nacional de 2400 mexicanos mayores de 18 años del 27 de septiembre al 20 octubre de 2010. Como se

${ }^{26}$ http:/ / lasamericasyelmundo.cide.edu/

${ }^{27}$ La encuesta fue levantada en 2010-2011 por equipos nacionales en Brasil, Colombia, Ecuador y Perú. Desafortunadamente, los cuestionarios no son completamente homogéneos (por ejemplo, en Brasil no se incluyeron preguntas sobre migración y en Colombia sólo se hicieron algunas sobre emigración). 
mencionó antes, hay muy pocas encuestas nacionales para estudiar las actitudes hacia los inmigrantes en los países en desarrollo. Esta encuesta es más útil que cualquier recurso de otra encuesta, ya que contiene preguntas sobre inmigrantes de diferentes nacionalidades. Esto permite un examen detallado tanto de los grupos potenciales de xenofobia y xenofilia como de la competencia en el mercado laboral.

Para las variables dependientes, hemos seleccionado cuatro preguntas que reflejan las actitudes y opiniones de los mexicanos sobre el volumen de extranjeros y sobre los diferentes grupos de extranjeros que viven en el país. En primer lugar, número es una variable dicotómica en que el valor 1 es dado a aquellos que piensan que hay demasiados extranjeros. Alrededor de un tercio de los encuestados piensa que hay de hecho demasiados extranjeros viviendo en México. En segundo lugar, utilizamos la opinión general de los mexicanos sobre los extranjeros que ya viven en el país, en contraste a la estimación sobre su número. A pesar de que la encuesta en 2010 no incluyó una pregunta específica acerca de las actitudes hacia los inmigrantes altamente cualificados y poco cualificados, ${ }^{28}$ hemos utilizado las opiniones de los encuestados acerca de los estadounidenses y guatemaltecos para capturar el hecho de que los mexicanos pueden tener opiniones diferentes sobre diferentes nacionalidades en función de sus cualificaciones, habilidades y rasgos étnicos. Sobre la base de la discusión anterior, se utiliza la opinión de los encuestados acerca de los estadounidenses como una representación (relativa) de las actitudes hacia los inmigrantes altamente calificados y la opinión de los guatemaltecos como un indicador de las actitudes hacia los inmigrantes poco calificados.

Estas últimas tres variables van de 1 (muy mala opinión) a 5 (muy buena opinión). ${ }^{29}$ Resulta interesante que el porcentaje de

${ }^{28}$ Los levantamientos de 2012 y 2014-2015 de la encuesta Las Américas y el Mundo sí incluyó en Argentina, Brasil, Chile, Colombia, Ecuador, México y Perú un experimento concreto para estimar las diferencias de las actitudes hacia los inmigrantes considerando si son altamente o poco calificados. Estos resultados serán analizados en un artículo posterior.

${ }^{29}$ En estos casos la pregunta utilizada fue: "Y ahora dígame, ¿cuál es su opinión sobre los siguientes grupos de extranjeros que viven en México: muy buena, buena, 
las personas que respondieron tener una mala opinión sobre los estadounidenses (19.8\%) es mayor que el porcentaje de las que respondieron tener una mala opinión de los inmigrantes en general $(10.7 \%)$. Los resultados son aún peores para los guatemaltecos $(28.5 \%)$.

Teniendo en cuenta que número es una variable dependiente binaria, se estimó un conjunto de modelos probit. Para las cinco categorías de opinión, estadounidenses y guatemaltecos se utilizaron modelos de regresión lineal. Todas las estimaciones han sido ponderadas para tener en cuenta las estratificaciones por sexo, edad y región.

En cuanto a las variables explicativas, para probar las hipótesis de competencia en el mercado laboral hemos seguido los últimos trabajos sobre el tema. ${ }^{30}$ En primer lugar, hemos utilizado la $e d u$ cación como una medida de las cualificaciones individuales: esta variable cuenta el número total de años completos de educación formal del entrevistado. ${ }^{31}$

En línea con los estudios recientes, hemos utilizado una medida alternativa de la competencia en el mercado laboral. De acuerdo con la situación laboral del encuestado, usamos una variable dicotómica que mide si el encuestado está dentro o fuera de la fuerza de trabajo (trabajo $=1$ ). En teoría, los primeros son más vulnerables a enfrentar la competencia en el mercado laboral y, por lo tanto, deben mostrar una actitud más restrictiva. Además, para estimar la última hipótesis económica alternativa, hemos incluido la opinión del entrevistado sobre si los extranjeros contribuyen a la economía nacional (contribución) ${ }^{32}$ y el ingreso subjetivo

mala o muy mala?", en donde se incluyeron a estadounidenses y guatemaltecos entre estos grupos.

${ }^{30}$ Hainmuller y Hiscox, art. cit.; Hainmuller y Hiscox, art. cit.; Mayda, art. cit.; Scheve y Slaughter, art. cit.

${ }^{31}$ Para este indicador se utilizó la pregunta: “¿Hasta qué año escolar estudió usted (grado máximo)?”. Las respuestas van de "ninguno" hasta "posgrado".

32 Con el propósito de contrastar las reacciones individuales a diferentes categorías de respuestas, el diseño de la encuesta dividió la muestra nacional en dos sub-muestras. Sin embargo, nosotros utilizamos la muestra completa incorporando dos preguntas en una sola variable. A la mitad de los encuestados se les preguntó: 
de los encuestados (evaluación del encuestado del ingreso de su hogar). ${ }^{33}$

Como se puso de manifiesto en la sección 3, las opiniones acerca de la inmigración se explican probablemente por predisposiciones sociales, tales como las actitudes hacia determinados grupos de diferente origen étnico y país. La exploración de opiniones sobre dos grupos de inmigrantes (estadounidenses en contraste con guatemaltecos) no sólo contribuye a explorar cómo los inmigrantes con diferentes cualificaciones son considerados, sino también si los diferentes antecedentes históricos y étnicos pueden ser de importancia. Teniendo en cuenta esta relación histórica, los puntos de vista de los mexicanos acerca de la difusión de ideas y costumbres extranjeras (cosmopolitismo) ${ }^{34}$ y los sentimientos nacionalistas (nacionalismo ${ }^{35}$ tienen amplias probabilidades de desempeñar un papel

\footnotetext{
"En general, ¿qué tan de acuerdo está usted con las siguientes afirmaciones sobre los extranjeros que viven en México? Los extranjeros que viven en México contribuyen a la economía mexicana. ¿Diría usted que está muy de acuerdo, algo de acuerdo, algo en desacuerdo o muy en desacuerdo?”. En este caso codificamos las respuestas de la siguiente manera: 0 significa "muy en desacuerdo" y "algo en desacuerdo" y 1 significa "algo de acuerdo" y "muy de acuerdo". A la otra mitad de los encuestados se les preguntó: "Ahora, usando la misma escala de 1 a 7 donde 1 es TOTALMENTE EN DESACUERDo, 7 es TOTALMENTE DE ACUERdo quiero que me diga ¿qué tan de acuerdo está usted con las siguientes afirmaciones sobre los extranjeros que viven en México? Los extranjeros que viven en México contribuyen a la economía mexicana". En este caso construimos una variable dicotómica de la siguiente manera: aquellos que respondieron 1, 2 y 3 se les asignó un 0 (es decir "desacuerdo") y aquellos que respondieron 4, 5, 6 y 7 se les asignó un 1 (es decir "acuerdo"). Probamos si existían diferencias en la distribución de las respuestas con las dos escalas de respuestas y los resultados mostraron que, estandarizando las respuestas entre 0 y 1 , los dos grupos encuestados tienen la misma media de respuestas.

${ }^{33}$ La pregunta concreta fue: "Con el total del ingreso familiar, diría usted que..." y las respuestas fueron codificadas así: 1 = "No les alcanza y tienen grandes dificultades", 2 = "No les alcanza y tienen dificultades", 3 = "Les alcanza justo, sin grandes dificultades" y 4 = "Les alcanza bien y pueden ahorrar".

${ }^{34}$ La pregunta concreta fue: "Para usted, ¿es bueno o malo que las ideas y costumbres de otros países se difundan en México?” y las respuestas fueron codificadas como 0 = "malo" y 1 = "bueno". Según los resultados, $50 \%$ de los mexicanos piensa que la difusión de ideas extranjeras es algo bueno.

${ }^{35}$ La pregunta concreta fue: “¿Qué tan orgulloso está usted de ser mexicano?" y las respuestas se dicotomizaron de la siguiente manera: 0 = "nada orgulloso"
} 
relevante. Le dimos un valor de 1 a los encuestados que reciben las ideas y hábitos extranjeros como positivos y a aquellos que presentan un fuerte orgullo nacional. Aproximadamente la mitad de los encuestados da la bienvenida a ideas y costumbres extranjeras, mientras que una abrumadora mayoría declara estar algo o muy orgulloso de ser mexicano. Además, como establecimos en las hipótesis sociales, es importante estimar la evaluación del entrevistado del nivel de amenaza de los inmigrantes a la seguridad pública (seguridad). ${ }^{36}$ La variable tiene un valor de 1 si los encuestados están en desacuerdo con que los inmigrantes causan problemas de seguridad. Alrededor de la mitad está en desacuerdo o muy en desacuerdo con la afirmación de que los inmigrantes son una amenaza para la seguridad.

El caso mexicano es interesante y peculiar por varias razones, entre ellas, su patrón de migración internacional a través de la emigración y como país transmigratorio. Teniendo en cuenta esta peculiaridad, se emplearon dos indicadores de la experiencia de los ciudadanos con flujos migratorios. En primer lugar, consideramos si

y "poco orgulloso" y 1 = "algo orgulloso" y "muy orgulloso". Ejecutamos los modelos sin hacer dicotómica la variable y los resultados fueron prácticamente los mismos.

${ }^{36}$ Como en la pregunta sobre la contribución de los extranjeros a la economía, hemos incorporado a las dos sub-muestras en una sola pregunta. A la mitad de los encuestados se les preguntó: "En general, ¿qué tan de acuerdo está usted con las siguientes afirmaciones sobre los extranjeros que viven en México? Los extranjeros que viven en México generan inseguridad. ¿Diría usted que está muy de acuerdo, algo de acuerdo, algo en desacuerdo o muy en desacuerdo?”. En este caso codificamos las respuestas de la siguiente manera: 1 significa "muy en desacuerdo" y "algo en desacuerdo" y 0 significa "algo de acuerdo" y "muy de acuerdo". A la otra mitad de los encuestados se les preguntó: "Ahora, usando la misma escala de 1 a 7 donde 1 es totalmente en Desacuerdo, 7 es totalmente de acuerdo quiero que me diga ¿qué tan de acuerdo está usted con las siguientes afirmaciones sobre los extranjeros que viven en México? Los extranjeros que viven en México generan inseguridad". En este caso construimos una variable dicotómica de la siguiente manera: aquellos que respondieron 1, 2 y 3 se les asignó un 1 (es decir "desacuerdo") y aquellos que respondieron 4, 5, 6 y 7 se les asignó un 0 (es decir "acuerdo"). También probamos si existían diferencias en la distribución de las respuestas con las dos escalas de respuestas y los resultados mostraron que, estandarizando las respuestas entre 0 y 1 , los dos grupos encuestados tienen la misma media de respuestas. 
el individuo tiene contacto personal con los extranjeros que viven en el país (contacto). Con base en resultados anteriores, ${ }^{37}$ esperamos que el contacto directo o la relación con los extranjeros influyan positivamente en sus actitudes hacia este grupo. Sin embargo, sólo $18 \%$ de los encuestados afirma tener contacto con los inmigrantes. En segundo lugar, se incluyó una variable para captar si el encuestado tiene familiares residentes en el exterior (familiares en el extranjero). Alrededor de la mitad de la muestra respondió afirmativamente a esta pregunta. Estamos interesados en saber si tener familiares en el extranjero moldea de alguna manera la opinión de los encuestados sobre los inmigrantes y la política de inmigración.

Todos los modelos incluyen otra serie de variables de control. Estas son las variables socio-demográficas estándares: género (hombre) y edad (medida en años). También como variable de control se incluyó la orientación política de los individuos (ideología) medida a lo largo de una escala de diez puntos ideológicos. ${ }^{38}$ Por último, controlamos con dos indicadores la ubicación geográfica de los encuestados. En primer lugar, los inmigrantes en México tienden a concentrarse en ciertas regiones, con una gran actividad en las fronteras norte y sur (muchos estadounidenses se establecen en los estados del norte de México y los migrantes centroamericanos en el sur de México). Se utilizó un indicador regional para dar cuenta de este hecho (norte, sur) ${ }^{39}$ En segundo lugar, se utiliza una variable para distinguir si los encuestados viven en localidades rurales o urbanas ( urbano $=1$ ). Teniendo en cuenta el hecho de que la mayoría de los inmigrantes se concentra en las zonas urbanas, ${ }^{40}$ y que de acuerdo con los modelos de análisis de la zona de

${ }^{37}$ McClare, art. cit.; Sides y Citrin, art. cit.

${ }^{38}$ Usamos la pregunta: "Hablando de su orientación política, ¿dónde se ubica usted en una escala de 0 a 10, donde 0 significa políticamente de izquierda y 10 políticamente de derecha?”. Usamos esta auto-ubicación en la escala ideológica con los valores de $0=$ izquierda a $10=$ derecha.

${ }^{39}$ En la edición de 2010 de la encuesta se incorporaron sobre-muestras regionales con el propósito de tener inferencias válidas no sólo para el país en conjunto, pero también para regiones (norte y sur). Estos permitió estar más seguros de las estimaciones de estas variables regionales.

${ }^{40}$ Rodríguez, op. cit. 
concentración de migrantes esto podría aumentar los temores de la competencia en el mercado laboral, se prevé que los encuestados que viven en estos lugares tengan puntos de vista menos liberales con respecto a la política de inmigración. En la muestra, el $18 \%$ vive en la región del norte, mientras que el $72 \%$ vive en zonas urbanas.

\section{Resultados}

En el cuadro 1 presentamos los modelos generales para estimar nuestras cuatro variables dependientes. Estos resultados se pueden resumir de la siguiente manera. Recordemos que la principal variable independiente para capturar los temores de la competencia del mercado de trabajo es la educación, entonces, si los miedos de la competencia del mercado laboral persisten, los encuestados más educados van a ser más propensos a pensar que hay demasiados inmigrantes y a tener opiniones desfavorables. ${ }^{41}$ Así, en primer lugar, aparte de explicar la opinión general que los mexicanos tienen de los extranjeros y su opinión acerca de los guatemaltecos, la educación no es estadísticamente significativa en ningún otro modelo. Contrario a las expectativas, los más educados tienen una mejor opinión de los inmigrantes en general, salvo en el caso de los guatemaltecos. Por lo tanto, parece que los intereses económicos y los temores de la competencia en el mercado laboral a partir de los niveles de educación individuales son poco relevantes. Sin embargo, si tenemos en cuenta los puntos de vista socio-trópicos de la economía (contribución), nos encontramos que aquellos que

${ }^{41}$ Es posible que los encuestados más educados también sean más cosmopolitas, que tengan mayor contacto con extranjeros, sean menos nacionalistas y reconozcan más fácilmente la contribución económica que hacen los extranjeros al país. En ese sentido, explícitamente controlamos estas variables que capturan esos rasgos individuales. Al hacerlo, podemos estar casi seguros de que la educación indica el nivel de las habilidades del encuestado, en vez de ser un efecto de otra disposición actitudinal altamente correlacionada con la educación. Sorprendentemente, las correlaciones en nuestros datos son bajas. El coeficiente de correlación más alto fue de $0.26(\mathrm{p}<0.05)$ entre años de educación y tener contacto con inmigrantes. 
aceptan que los extranjeros contribuyen a la economía nacional tienen mejores opiniones de los inmigrantes en general y, en particular, de los ciudadanos estadounidenses y guatemaltecos. Por último, los que consideran que sus ingresos familiares son buenos mantienen una mejor opinión de los extranjeros.

En segundo lugar, en general los indicadores de preocupaciones sociales funcionan mejor, como se esperaba, cuando se pretende explicar las opiniones hacia los inmigrantes. Hay algunos resultados que son muy robustos e interesantes. Para empezar, los más cosmopolitas (o menos etnocéntricos), es decir, los más favorables a la llegada de nuevas ideas, tienen una mayor probabilidad de pensar que hay pocos extranjeros, por lo que tienen mejores opiniones de los inmigrantes en general y de los dos grupos de inmigrantes en particular. Los problemas de seguridad no siempre influyen en las actitudes: aquellos en desacuerdo con la opinión de que los inmigrantes son un problema de seguridad real son más propensos a pensar que los extranjeros son pocos y a tener mejores opiniones de los inmigrantes en general y de cada uno de los grupos en particular. Por último, los hombres tienen una mejor opinión de los inmigrantes que las mujeres.

En cuanto a la concentración geográfica, los que viven en la región del norte tienen mejor opinión de los inmigrantes en general y de los inmigrantes estadounidenses que las personas que viven en otras regiones (norte). La concentración de inmigrantes probablemente aumenta los temores de la competencia en el mercado laboral. Esta es probablemente la razón por la cual en zonas urbanas (urbano) es menos probable que los encuestados piensen que hay pocos extranjeros en el país.

La presentación visual de los resultados puede ayudar al lector a entender la dirección y la magnitud de los efectos reportados en el cuadro 1. Dado el carácter no lineal del primer modelo, los coeficientes dan poca información acerca del efecto que las diferentes variables tienen en la probabilidad de pensar que hay demasiados extranjeros en el país. En ese sentido, la figura 1 grafica el efecto marginal de cada una de las variables en la variable número y el parámetro estimado para la variable opinión midiendo las actitudes hacia los migrantes en general, junto con el $95 \%$ de confianza de 
los intervalos. Cuanto más lejos esté el punto estimado de la línea cero, mayor el efecto que tendrá la variable independiente sobre la variable dependiente. El 95\% de confianza de los intervalos indica si los efectos son significativos o no.

\section{Cuadro 1}

Determinantes de las actitudes hacia los inmigrantes en México

\begin{tabular}{|c|c|c|c|c|}
\hline & Número & Opinión & Estadounidenses & Guatemaltecos \\
\hline \multicolumn{5}{|l|}{$\begin{array}{l}\text { Explicaciones } \\
\text { económicas }\end{array}$} \\
\hline Educación & $0.011(0.009)$ & $0.013(0.005)^{* *}$ & $-0.001(0.007)$ & $-0.012(0.007)^{*}$ \\
\hline Contribución & $-0.010(0.08)$ & $0.253(0.05)^{* * *}$ & $0.393(0.06)^{* * *}$ & $0.174(0.06)^{* *}$ \\
\hline Ingreso & $-0.024(0.04)$ & $0.079(0.02)^{* * *}$ & $0.026(0.03)$ & $0.066(0.03)^{*}$ \\
\hline \multicolumn{5}{|l|}{$\begin{array}{l}\text { Explicaciones } \\
\text { sociales }\end{array}$} \\
\hline Cosmopolitismo & $0.174(0.06)^{* * *}$ & $0.400(0.04)^{* * *}$ & $0.238(0.04)^{* * *}$ & $0.143(0.05)^{* * *}$ \\
\hline Nacionalismo & $-0.431(0.14)^{* * *}$ & $0.120(0.09)$ & $0.035(0.107)$ & $-0.007(0.11)$ \\
\hline Ideología política & $0.025(0.01)^{* * *}$ & $-0.0003(0.007)$ & $-0.006(0.009)$ & $-0.005(0.01)$ \\
\hline Seguridad & $0.373(0.06)^{* * *}$ & $0.105(0.04)^{* *}$ & $0.065(0.04)^{* *}$ & $0.124(0.05)^{* *}$ \\
\hline $\begin{array}{l}\text { Familiares en } \\
\text { extranjero }\end{array}$ & $0.119(0.06)^{*}$ & $-0.071(0.04)^{*}$ & $0.047(0.04)$ & $-0.077(0.05)$ \\
\hline Contacto & $0.105(0.08)$ & $0.116(0.05)^{* *}$ & $0.088(0.06)$ & $-0.072(0.06)$ \\
\hline Hombre & $0.193(0.06)^{* * *}$ & $0.039(0.04)$ & $0.049(0.04)$ & $0.066(0.05)$ \\
\hline Edad & $-0.002(0.06)$ & $-0.002(0.001)^{*}$ & $-0.004(0.002)^{* *}$ & $-0.008(0.00) * * *$ \\
\hline Norte & $0.063(0.09)$ & $0.144(0.05)^{* * *}$ & $0.359(0.06)$ & \\
\hline Sur & & & & $-0.044(0.08)$ \\
\hline Urbano & $-0.191(0.08)^{* *}$ & $-0.060(0.04)$ & $0.025(0.05)$ & $-0.076(0.06)$ \\
\hline Constante & $-0.394(0.24)$ & $2.853(0.14)^{* * *}$ & $2.874(0.17)^{* * *}$ & $3.157(0.187)^{* * *}$ \\
\hline Observaciones & 1489 & 1703 & 1665 & 1527 \\
\hline R2 & 0.043 & 0.119 & 0.078 & 0.036 \\
\hline
\end{tabular}

Notas: Errores típicos entre paréntesis. Significación estadística: $* \mathrm{p}<0.10 ; * * \mathrm{p}<0.05$; $* * * \mathrm{p}<0.01$ 
Como muestra además la figura 1, ser cosmopolita, hombre, vivir en el norte y, sobre todo, no considerar a los inmigrantes como un problema de seguridad reduce la probabilidad de pensar que hay demasiados inmigrantes en el país. En cuanto a la opinión general acerca de los inmigrantes, todas las variables anteriores contribuyen a tener una mejor opinión de ellos. Nótese que también considerar que los inmigrantes contribuyen a la economía y tener contacto con extranjeros mejora la opinión.

\section{FigurA 1}

Efectos marginales y parámetros de los modelos para explicar las actitudes hacia los inmigrantes en México
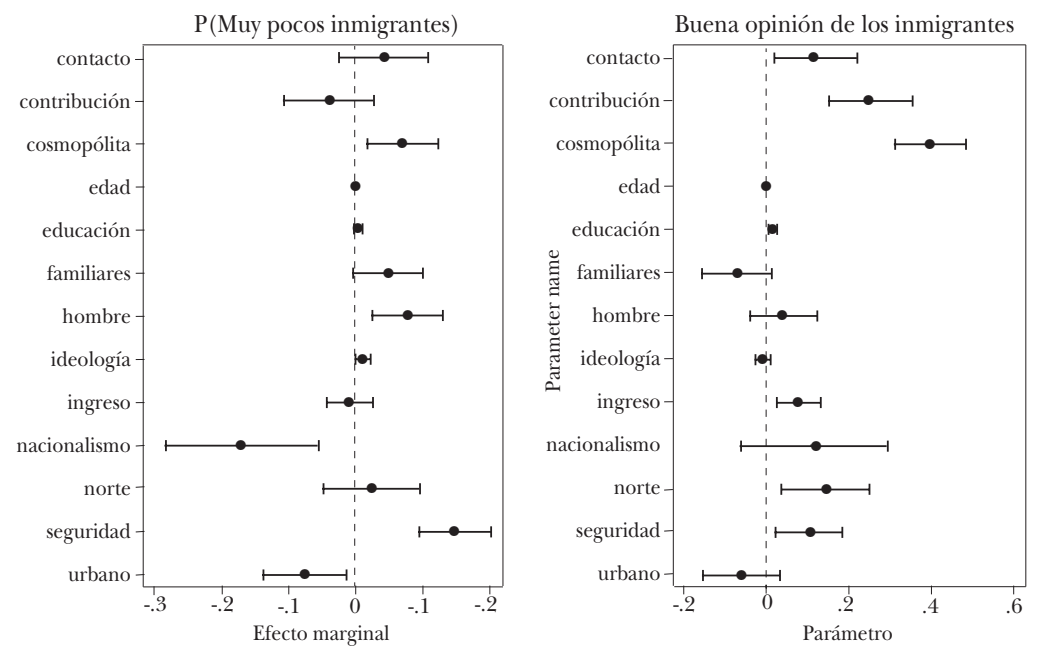

Por su parte, la figura 2 muestra que casi no hay diferencias entre las variables que afectan las opiniones acerca de los estadounidenses y guatemaltecos. Los individuos cosmopolitas y aquellos que piensan que los extranjeros contribuyen a la economía y que no amenazan la seguridad nacional mantienen una mejor opinión de ellos. Aunque hay diferencias entre la magnitud de los efectos para los dos grupos de inmigrantes. Por ejemplo, 
pensar que los inmigrantes contribuyen a la economía mejora la opinión acerca de los residentes estadounidenses en México por 0.39 puntos mientras que mejor para los guatemaltecos por 0.17 puntos. Resulta interesante que estar abierto a ideas extranjeras mejora la opinión de los estadounidenses por 0.23 puntos. En contraste, el incremento equivalente para los guatemaltecos es de 0.14. Esto dice que la receptividad a las ideas extranjeras y de costumbres incide en las opiniones de los estadounidenses y guatemaltecos de forma diferente. Cabe señalar también que, fuera de las variables actitudinales, encontramos que entre más educados es menos probable mantener una buena opinión de los guatemaltecos, aunque el efecto sustancial de esta variable es muy pequeño. Tomados en conjunto, estos resultados son indicativos de que las preocupaciones etnocéntricas están aún vivas y apunta a una especie de prejuicio grupal en contra de los guatemaltecos.

\section{Figura 2}

Parámetros de los modelos para explicar las actitudes hacia los estadounidenses y guatemaltecos en México
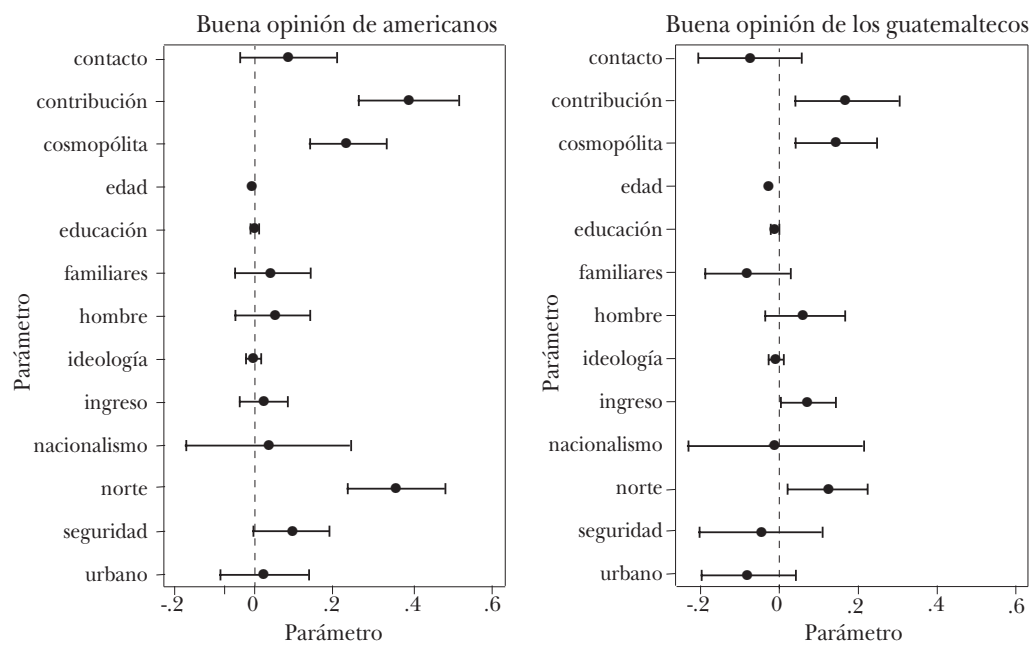
Finalmente, en el cuadro 2 mostramos los resultados sólo para las variables dependientes número y opinión, dividiendo la muestra entre aquellos que están en el mercado laboral (los entrevistados que reportaron estar empleados) y aquellos fuera del mercado laboral (cualquier otra respuesta a la pregunta sobre ocupación). Como se estableció antes, la educación de aquellos que sí están en el mercado laboral debería producir más claramente que los extranjeros son muchos y a tener opiniones menos positivas que entre aquellos fuera del mercado laboral. No obstante, como vemos en los resultados, la educación sigue sin ser estadísticamente significativa para explicar las actitudes hacia inmigrantes; de hecho el único resultado significativo ocurre entre quienes no están en el mercado laboral: a más años de educación, mejor opinión sobre los extranjeros. Pensar que los extranjeros contribuyen a la economía es una variable significativa, como en los modelos anteriores. Y finalmente, el nivel de ingresos es importante, de nuevo, sólo entre aquellos fuera del mercado laboral. En resumen, tampoco la situación en el mercado laboral parece sostener que los individuos se sientan amenazados por los inmigrantes.

\section{CuAdro 2}

Determinantes de las actitudes hacia los inmigrantes en México. Estatus en el mercado laboral y ocupación

\begin{tabular}{|c|c|c|c|c|}
\hline & \multicolumn{2}{|c|}{ En el mercado de trabajo } & \multicolumn{2}{|c|}{ Fuera del mercado de trabajo } \\
\hline & Número & Opinión & Número & Opinión \\
\hline \multicolumn{5}{|l|}{$\begin{array}{l}\text { Explicaciones } \\
\text { económicas }\end{array}$} \\
\hline Educación & $0.049(0.01)$ & $0.059(0.007)$ & $0.039(0.01)^{* *}$ & $0.026(0.009)^{* * *}$ \\
\hline Contribución & $-0.287(0.11)^{* *}$ & $0.303(0.06)^{* * *}$ & $0.188(0.14)$ & $0.183(0.08)^{* *}$ \\
\hline Ingreso & $-0.078(0.05)$ & $0.099(0.03)^{* * *}$ & $-0.048(0.07)$ & $0.055(0.043)$ \\
\hline Constante & $-0.063(0.31)$ & $2.929(0.18)^{* * *}$ & $-1.185(0.43) * * *$ & $2.607(0.25)^{* * *}$ \\
\hline Observaciones & 870 & 993 & 617 & 708 \\
\hline $\mathrm{R} 2$ & 0.054 & 0.127 & 0.053 & 0.122 \\
\hline
\end{tabular}

Notas: Errores típicos entre paréntesis. Significación estadística: * $\mathrm{p}<0.10 ; * * \mathrm{p}<0.05$; $* * * \mathrm{p}<0.01$. En estos modelos se incluyeron todas las otras variables de los modelos del Cuadro 1 (cosmopolitanismo, nacionalismo, ideología política, seguridad, familiares en el extranjero, contacto, edad, hombre, norte, urbano), pero por economía del espacio no se presentan. Están disponibles en consulta a petición. 
Un último comentario refiere a otros resultados negativos, esto es, a explicaciones teóricas que no están apoyadas por los datos. Primero, el nacionalismo no afecta la perspectiva acerca de los inmigrantes o sus números. La ideología no parece tampoco explicar las actitudes con respecto a la inmigración. Aunque México es un país de emigración, tener familiares en el exterior tampoco parece ser relevante en cambiar las opiniones acerca de los inmigrantes. Finalmente, a pesar de la concentración de inmigrantes en entornos urbanos y los miedos consecuentes de competencia que surgen por esta concentración, los residentes urbanos no eran más probables de tener una opinión anti-inmigrante que los residentes no-urbanos.

\section{Comentarios Finales y AGENDA DE INVESTigación}

Si México nunca se ha enfrentado a fuertes presiones de inmigración -y de hecho, la población inmigrante apenas alcanza el $1 \%$ de la población-, ¿por qué $30 \%$ de los mexicanos creen que hay demasiados inmigrantes? A la luz de los factores que han dado forma a la política de inmigración de México a lo largo del tiempo y con base en la investigación existente sobre los determinantes de las actitudes hacia los inmigrantes, contemplamos dos hipótesis alternativas. El primer conjunto de hipótesis hizo hincapié en los temores de la competencia en el mercado laboral y el segundo consideró las preocupaciones sociales. En definitiva, nos encontramos con muy poco apoyo para las variables de competencia en el mercado laboral: los niveles educativos de los encuestados y el estatus del mercado laboral no son significativos en explicar las perspectivas del público mexicano acerca de los inmigrantes y sus números. Y cuando ellos son significativos marginalmente (ocupación de los encuestados), estas variables no funcionan como la teoría lo predice, sugiriendo una percepción errónea acerca del promedio de los niveles de habilidades y ocupaciones de los extranjeros.

Por el contrario, nuestros resultados ponen de relieve el conjunto de hipótesis de "explicaciones sociales". Aquellos que son más 
etno-céntricos y que consideran a los inmigrantes como una amenaza a la seguridad mantienen las peores opiniones de los inmigrantes y son más propensos a pensar que su número es muy alto. Los efectos son robustos a las diferentes especificaciones de los modelos; y ellos exhiben el mayor impacto en las variables dependientes. De manera interesante, estas variables afectan las diferencias entre las percepciones de los estadounidenses y guatemaltecos, sugiriendo que las preocupaciones etno-céntricas y el prejuicio en contra de grupos específicos de migrantes persisten.

Concluimos este trabajo con algunas reflexiones acerca de lo que añaden nuestros resultados a los debates teóricos y empíricos sobre los determinantes de las actitudes hacia los inmigrantes y la inmigración. En un excelente artículo de revisión, Ceanobu y Escandell $^{42}$ hacen varias observaciones sobre el estado actual de la investigación sobre las actitudes hacia la inmigración y los inmigrantes en paralelo a la proliferación de datos de encuestas multinacionales. Los autores señalan, con razón, que hay una excesiva concentración de investigación en países avanzados y que apenas se ha prestado atención a la forma en que la opinión pública en mercados emergentes y en desarrollo ve a la inmigración en sus regiones. Teniendo en cuenta que existen flujos importantes de inmigrantes que ocurren entre países Sur-Sur, el esfuerzo para recopilar datos fuera del mundo desarrollado no debe aplazarse. Por otra parte, algunos mercados emergentes están comenzando a participar activamente en el mercado internacional de inmigrantes cualificados. Con la situación económica en países avanzados sometida a cierta presión, los flujos de inmigrantes hacia el sur pueden comenzar a aumentar en magnitud.

México es un caso bastante peculiar, ya que el mayor grupo de extranjeros que llega es de un país desarrollado que, a su vez, es el principal destino de los emigrantes mexicanos. También es una ruta muy importante para los transmigrantes en su camino a los Estados Unidos. Para entender los determinantes de las actitudes hacia los inmigrantes, nos basamos en predictores existentes a nivel individual que capturan los intereses económicos y no

${ }^{42}$ Ceanobu y Escandell, art. cit. 
económicos. Y, dado que se trata de un solo país, nos basamos en la historia para poner el caso en un marco contextual. A pesar de las especificidades de México, algunos de nuestros hallazgos están claramente alineados con el conocimiento acumulado en los países avanzados: ${ }^{43}$ las actitudes sociales y no los intereses económicos son mejores predictores para explicar las actitudes hacia los inmigrantes. A diferencia de los países avanzados, la educación tiene poca importancia en explicar las opiniones acerca de los inmigrantes. De forma sorprendente, parece que la mayor aceptación de diversidad que es frecuentemente asociada con niveles de educación más alto no opera en el contexto mexicano.

$\mathrm{Al}$ igual que en los países avanzados, la mayor parte de la efectividad para entender las opiniones hacia los inmigrantes y la inmigración ocurre en el ámbito de los predictores de actitudes. Creemos que mientras en los países avanzados las preocupaciones etnocéntricas surgen del efecto de las minorías étnicas de los inmigrantes en sociedades relativamente homogéneas, en países en vías de desarrollo un objetivo de las políticas de inmigración ha sido buscar que las sociedades etnicamente heterogéneas se vuelvan más homogéneas mediante la selección de grupos particulares de inmigrantes. El caso de México es indicativo y, probablemente, está lejos de ser único. Estas diferencias tendrán que tomarse en cuenta en investigaciones futuras. Por último, mientras que las bases de datos con encuesta multinacionales y comparativas pueden ser esclarecedoras para aprovechar la relevancia de los diferentes contextos nacionales, y que son muy necesarias fuera de los países avanzados, no cabe duda de que los datos de la encuesta multinacional tendrán que ser complementados con estudios de caso en profundidad para incorporar legados históricos y comprender qué hay detrás de ciertas correlaciones estadísticas.

${ }^{43}$ Sides y Citrin, art. cit.; Hainmueller y Hiscox, art. cit.; Hainmueller y Hiscox, art. cit. 


\section{Bibliografía}

Arias, Juan, "Brasil busca convertirse en un país de inmigrantes profesionales”, El País, 15 de enero de 2012.

Basave, Agustín, Análisis del nacionalismo mexicano en torno a la mestizofilia de Andrés Molina Enríquez, México, FCE, 2002.

Borjas, George, Heaven's Door: Immigration Policy and the American Economy, Princeton, Princeton University Press, 1999.

Borjas, George, Richard Freeman y Lawrence Katz, "Searching for the Effect of Immigration on the Labor Market", American Economic Review, vol. 86, núm. 2, 1996, pp. 247-251.

, "How Much Do Immigration and Trade Affect Labor Market Outcomes?”, Brooking Papers on Economic Activity, vol. 1, 1997, pp. 1-90.

Chandler, Charles y Yung-Mei Tsai, "Social Factors Influencing Immigration Attitudes: An Analysis of Data from the General Social Survey", Social Science Journal, vol. 38, núm. 2, 2001, pp. 177-188

Ceobanu, Alin y Xavier Escandell, "Comparative Analysis of Public Attitudes Toward Immigrants and Immigration Using Multinational Survey Data: A Review of Theories and Research", Annual Review of Sociology, vol. 36, 2010, pp. 309-328.

Citrin, Jack, Donald Green, Christopher Muste y Cara Wong, "Public Opinion toward Immigration Reform: The Role of Economic Motivations”, Journal of Politics, vol. 59, 1997, pp. 858-881.

Citrin, Jack y John Sides, "Immigration and the Imagined Community in Europe and the United States", Political Studies, vol. 56, 2008, pp. 33-56.

Comisión Nacional de Derechos Humanos (CNDH), Informe especial sobre secuestro de migrantes en México, México, 2011.

Cobo, Salvador, "Los inmigrantes españoles contemporáneos en México: Una mirada a sus perfiles sociodemográficos y sus patrones de participación laboral”, en Ernesto Rodríguez (ed.), Extranjeros en México. Continuidades y aproximaciones, México, Centro de Estudios Migratorios, 2006, pp. 173-198.

De Figueiredo, Rui y Zachary Elkins, "Are Patriots Bigots? An Inquiry into the Vices of In Group Pride”, American Journal of Political Science, vol. 47, 2003, pp. 171-188.

Faccini, Giovanni, Anna M. Mayda y Mariapia Mendola, "South-South 
Migration and the Labor Market: Evidence from South Africa”, documento presentado en la $8^{\text {th }}$ IZA Annual Migration Meeting and 3rd Migration Topic Week, Washington, 12-15 de mayo de 2011.

-, "What Drives Attitudes towards Immigrants in South Africa?", Centro Studi Luca D’Agliano, Development Studies Working Papers, núm. 325, 2011.

Fetzer, Joel S., Public Attitudes toward Immigration in the United States, France, and Germany, Nueva York, Cambridge University Press, 2000.

Florescano, Enrique, Historia de las historias de la nación mexicana, México, Taurus, 2002.

Gindling, Tim H., "South-South Migration: The Impact of Nicaraguan Immigrants on Earnings, Inequality and Poverty in Costa Rica”, World Development, vol. 37, núm. 1, 2009, pp. 116-126.

Gomes, Charles P., "La Migración labora en Brasil", en Ezquiel Texidó, Gladys Baer, Nora Pérez, Anna M. Santestevan y Charles P. Gomes, Migraciones laborales en Sudamérica: El Mercosur ampliado, Estudios sobre Migraciones Internacionales, Ginebra, International Labor Organization, 2003.

González Navarro, Moisés, Los extranjeros en México y los mexicanos en el extranjero, 1821-1970, vol. 1, México, El Colegio de México, 1993.

González, Guadalupe, Jorge Schiavon, David Crow y Gerardo Maldonado, México, las Américas y el Mundo 2010: Política exterior, opinión pública y líderes, México, cide, 2011.

Hainmueller, Jens y Michael Hiscox, "Educated Preferences: Explaining Attitudes toward Immigration in Europe", International Organization, vol. 61, 2007, pp. 399-442.

, "Attitudes toward Highly Skilled and Low-Skilled Immigration: Evidence from a Survey Experiment", American Political Science Review, vol. 104, 2010, pp. 61-84.

Kinder, Donald y Roderick Kiewiet, "Sociotropic Politics: The American Case”, British Journal of Political Science, vol. 2, 1981, pp.129-161.

Knight, Alan, "Peasants into Patriots: Thoughts on the Making of the Mexican Nation”, Mexican Studies/Estudios Mexicanos, vol. 10, núm. 1, 1994, pp. 135-161.

Lida, Clara E., 'Los españoles en el México independiente: 1821-1950. Un estado de la cuestión”, Historia Mexicana, vol. 2, núm, 222, 2006, pp. 613-650. 
Mayda. Anna M., "Who Is against Immigration? A Cross-Country Investigation of Individual Attitudes toward Immigrants", The Review of Economics and Statistics, vol. 88, 2006, pp. 510-530.

McLaren, Lauren, "Anti-Immigrant Prejudice in Europe: Contact, Threat Perception, and Preferences for the Exclusion of Migrants", Social Forces, vol. 81, núm 3, 2003, pp. 909-936.

Meyer, Lorenzo, "Estados Unidos y la evolución del nacionalismo defensivo mexicano", Foro Internacional, vol. 46, núm 3, 2006, pp. 421-464.

Mexico, las Américas y el Mundo. Encuesta 2010, México, cIDE, en http://lasamericasyelmundo.cide.edu

Misha, Prachi, "Emigration and wages in source countries: Evidence from Mexico", Journal of Development Economics, vol. 82, 2007, pp. 180-199.

Orcés, Diana M., "Democratic Values and Public Opinion toward Immigrants: The Case of Ecuador", Latin American Politics and Society, vol. 51, núm. 4, 2009, pp. 131-155.

Palma Mora, Mónica, De tierras extrañas: Un estudio sobre la inmigración en México, 1950-1990, México, InM-INAH-DGe Ediciones, 2008.

Pardos-Prado, Sergi, "Framing Attitudes toward Immigrants in Europe: When Competition Does Not Matter", Journal of Ethnic and Migration Studies, vol. 37, 2011, pp. 999-1015.

Paredes, Guillermo, "Migración de guatemaltecos a México y Estados Unidos a partir de la Encuesta sobre Migración en la Frontera Guatemala-México 2004: Un análisis de Estrategias Migratorias”, Migraciones Internacionales, vol. 5, núm. 1, 2009, pp. 93-124.

Peregil, Federico, "Argentina cambiará la ley para acoger a científicos españoles”, El País, 19 de febrero de 2012.

Pla Bruget, Dolores, "Ser español en México, para bien y para mal", en Delia Salazar (ed.), Xenofobia y xenofilia en la historia de México, siglos XIX y xx, México, Instituto Nacional de Migración/Instituto Nacional de Antropología e Historia/DGE Ediciones, 2006.

Ratha, Dilip y William Shaw, "South-South Migration and Remittances", World Bank Working Paper, núm. 102, 2007.

Rodríguez, Ernesto, "La inmigración en México a inicios del siglo xxi", en Ernesto Rodríguez (ed.), Extranjeros en México. Continuidades y aproximaciones, México, Centro de Estudios Migratorios, 2010, pp. 89-132. 
Salazar, Delia, "Introducción”, en Delia Salazar (ed.), Xenofobia y xenofilia en la historia de México, siglos XIX y XX, México, INM/INAH/DGE Ediciones, 2006.

Salazar, Delia, "Tres momentos de la inmigración internacional en México, 1880-1946”, en Ernesto Rodríguez (ed.), Extranjeros en México: Continuidades y aproximaciones, México, INM/ DGE Ediciones, 2010.

Scheve, Kenneth y Matthew Slaughter, "Labor Market Competition and Individual Preferences over Immigration Policy", The Review of Economics and Statistics, vol. 83, núm 1, 2001, pp. 133-145.

Sides, John y Jack Citrin, "European Opinion about Immigration: The Role of Identities, Interests, and Information", British Journal of Political Science, vol. 37, 2007, pp. 477-504.

Solimano, Andrés, International Migration in the Age of Crisis and Globalization. Historical and Recent Experiences, Nueva York, Cambridge University Press, 2010.

Vázquez, Josefina Z., Nacionalismo y educación en México, México, El Colegio de México, 1970.

Yankelevich, Pablo, ¿Deseables o inconvenientes? Las fronteras de la extranjería en el México posrevolucionario, México, Bonilla Artigas/Escuela Nacional de Antropología e Historia/Iberoamericana Vervuert, 2011. 